\title{
An Effective Hotspot Cell Management Scheme Using Adaptive Handover Time in 4G Mobile Networks
}

\author{
Dongwook Kim, Seunghak Lee, Hanjin Lee, and Hyunsoo Yoon \\ Dept. of EECS, Korea Advanced Institute of Science and Technology, Daejeon, Republic of Korea \\ Email: \{kimdw, shlee, hjlee, hyoon $\}$ camars.kaist.ac.kr
}

\begin{abstract}
We propose a hotspot cell management scheme using adaptive handover time in 4G mobile networks. 4G networks are expected to support various multimedia services over IP network and satisfy high spectral efficiency requirement with overcoming time and frequency selective fading due to the high speed of users. In cellular systems including 4G networks, hotspot cells can be occurred when available wireless resources at some location are not enough to sustain the needs of users. The hotspot cell can potentially lead to blocked and dropped calls and cause degradation of the service quality. In the meanwhile, a band of users enjoying multimedia services can move around in a 4G mobile network, which may generate heavy flows of traffic load in the network. This situation can generate the hotspot cell which has a short length of life at most a few minutes. In this paper, we propose a handover-based scheme which can effectively manage hotspot cells in 4G mobile networks. The proposed scheme adopts hard handoff mechanism and dynamically controls the time point of handover based on the amount of load of the current serving cell and the target cell. Adaptation of handover time according to the amount of load effectively and flexibly can manage the hotspot cell. And, through our hotspot cell management scheme, acceptable service quality can be supported as users maintain graceful connections with the network. In the simulation results, we find that our scheme makes smaller number of hotspot cells and supports higher service quality than compared schemes.
\end{abstract}

\section{INTRODUCTION}

Fourth generation(4G) mobile networks which will be a kind of future wireless communication systems aim at supporting various multimedia services with high data rate. To effectively provide multimedia services, the air interface technique should comply with packet-data transmission requirement. And, to support high data rate, spectral efficiency should be more enhanced than that of $3 \mathrm{G}$ cellular systems with overcoming time and frequency selective fading due to the high speed of users. OFDM(Orthogonal Frequency Division Multiplexing) is considered as one of the most compromising wireless techniques for future-generation cellular systems [1]. The OFDM transmission scheme, a special case of multicarrier transmission, is robust against frequency selective fading and narrowband interference [2]. Recently, a mobile

\footnotetext{
${ }^{1}$ This work was supported by the KOSEF (Korea Science and Engineering Foundation) through the AITrc (Advanced Information Technology Research Center) and the MIC(Ministry of Information and Communication), Korea, under the ITRC(Information Technology Research Center) support program supervised by the IITA(Institute of Information Technology Assessment)
}

system based on IP and OFDM is intensively considered as the next generation standard by $\mathrm{B} 3 \mathrm{G}$ (Beyond $3 \mathrm{G}$ ) or $4 \mathrm{G}$ committees such as IEEE 802.16e and IEEE 802.20 [3] [4].

In cellular systems, there can occur a situation that available wireless resources at some location are not enough to sustain the needs of users [5]. In a traffic accident on highway, a lot of traffic load can be generated in the cell which covers the accident place [6]. A cell which has heavy load than adjacent cells is referred to as hotspot cell. The hotspot cell can be analyzed by resource affordability, the ratio between the amount of available resources and the total amount of resources in a cell. If the resource affordability of a cell is less than or equal to a certain threshold, it is considered that the cell becomes the status of hotspot. In the hotspot cell, we assume that the resource affordability would be less than $20 \%$.

The hotspot cell problem degrades the service quality with leading to dropping of handover calls or blocking of new calls. And, the problem can cause degradation of resource utilization from the view point of overall system because available resources which remain in adjacent cells are not used. Consequently, the advantage of cellular system which allocates system resources by a cell unit for efficient usage may be disappeared due to the problem. The hotspot cell problem still remains as not being solved in $4 \mathrm{G}$ mobile networks.

In a $4 \mathrm{G}$ mobile network, a band of users enjoying multimedia services can move around in the overall network, which can generate heavy flows of traffic load. The cells which these flows pass through become the status of hotspot from a few seconds to a few minutes according to the speed of the band. That is, the hotspot cell which has a short length of life can be frequently generated in the network.

Many algorithms have been proposed to solve the hotspot cell problem. They can be classified by resource allocation scheme and load distribution scheme. In research area of resource allocation scheme, major papers proposed channel borrowing algorithms which utilize remained resources of lightly loaded cells [7] [8]. And, in research area of load distribution scheme, firstly, there are algorithms that control the transmitting power of the base station in order to distribute traffic load of the hotspot cell. ACS(Adaptive Cell Sizing) scheme in [9] is the representative algorithm which controls the transmitting power of the base station based on CDMA(Code Division Multiple Access) cellular system. Secondly, to distribute traffic 
load of the hotspot cell, handover-based algorithms which use functionalities of handover mechanism have been proposed. Among many algorithms, soft handover area resizing in [10] is referred to as the representative algorithm. [10] exploits features of soft handover functionality of CDMA cellular system in order to reduce load of the hotspot cell. With the scheme, the hotspot cell increases the threshold value, $T \_D R O P$ in order to reduce its soft handover area and if this threshold value is increased, the users who belong to the soft handover area are forcibly handed over the neighboring cells. The released resources in the hotspot cell are allocated to additional call requests with satisfying the total transmitting power constraints.

Previous works only focus on distribution of traffic load of the hotspot cell without considering the load status of neighboring cells. If neighboring cells are also heavily loaded, they can be also hotspot due to the traffic load distributed from the hotspot cell. And, the works only consider the situation that the status of hotspot is maintained for a quite long time. They may not effectively deal with the cell whose status becomes hotspot during at most a few minutes. In summary, if existing algorithms are applied to manage hotspot cells in $4 \mathrm{G}$ mobile networks, they may cause more hotspot cells in all the service area and may cause more deterioration of the service quality. Therefore, an effective hotspot cell management scheme suitable for $4 \mathrm{G}$ mobile networks is needed.

In this paper, we propose a handover-based scheme which can effectively manage hotspot cells in 4G mobile networks. 4G cellular systems will use OFDM as the physical layer technology and support hard handoff. Therefore, the proposed scheme which adopts hard handoff mechanism dynamically changes the time point of handover according to the load status of cells. The scheme can prevent the outbreak of hotspot cells within the network and enhance service quality. In the simulation, we can find that the proposed scheme generates smaller number of hotspot cells and supports higher satisfaction level of users than compared schemes.

The remainder of this paper is organized as follows. In Section II, we represent our scheme in details. And performance evaluation by comparison with other schemes is given in Section III. Finally, we conclude this paper in Section IV by summarizing and suggesting the future research directions.

\section{Adaptive Handover Time Scheme}

We present an adaptive handover time scheme for effective hotspot cell management in $4 \mathrm{G}$ mobile networks. Adaptive handover time means the time point of handover is dynamically changed according to the amount of traffic load of cells. The proposed scheme is based on hard handoff mechanism for cellular systems based on OFDM and MAHO(MobileAssisted HandOff) method. The scheme assumes that cells are connected through backbone network without restraints of the structure. The scheme is also expected not to impose high system complexity because the scheme only adopts

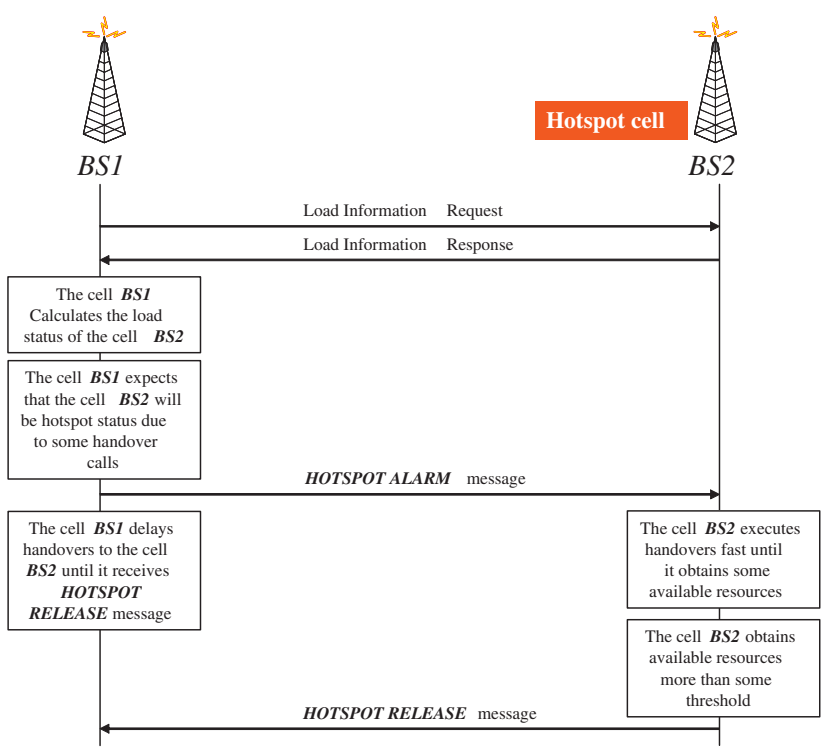

Fig. 1. Process of adaptive handover time scheme

hard handover parameters, hysteresis and absolute thresholds, without changing the mechanism itself. Conventional handover mechanism is based on degradation of the pilot signal strength of the base station. It does not consider the amount of load of the current serving cell and the target cell. If a handover is executed with using both signal strength and load information, base stations can recommend the best serving cell for users with guaranteeing high service quality.

Figure 1 shows the process of adaptive handover time scheme. As shown in Fig. 1, when the current serving cell, $B S 1$ receives the report from a mobile which includes signal strength and the signal strength is weaker than the specific threshold, $B S 1$ requests the load information of the target cell, $B S 2$. If $B S 1$ determines that $B S 2$ will become the status of hotspot due to increased load caused by handover calls, $B S 1$ sends the hotspot alarm message, HOTSPOT_ALARM to $B S 2$. And $B S 1$ delays all handovers caused by users who belong to the handover area between $B S 1$ and $B S 2$. BS2 recognizes that its status will be hotspot due to additional load caused by handover calls and reduces its load by executing all possible handovers earlier than scheduled in conventional handover mechanism. Through the fast handover execution, $B S 2$ can obtain available resources and prepare to handover calls occurred in the near future. If $B S 2$ gets out of the status of hotspot with fast handover execution and obtains the sufficient amount of available resources, $B S 2$ sends the hotspot release message, HOTSPOT RELEASE to BS1. If the amount of available resources are sufficient, it should meet Equation (1).

$$
N_{A}(S)>N_{\text {TOTAL }}(S) \cdot H_{d}+\alpha
$$

Where, $N_{A}(S)$ is the amount of available resources of the cell $S$ and $N_{\text {TOTAL }}(S)$ is the total amount of resources. We assume that the channel affordability of the hotspot cell is less 


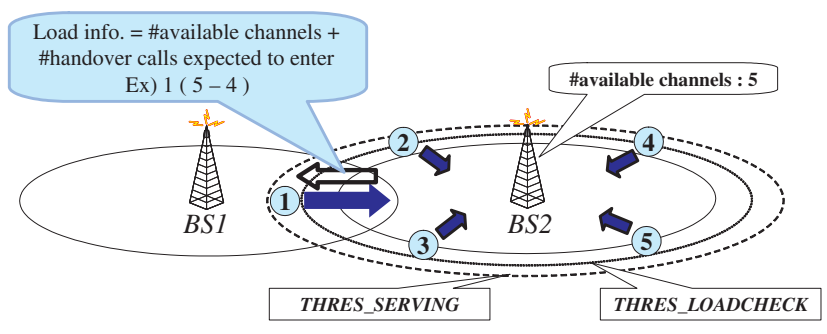

Fig. 2. Process of load calculation

than $H_{d}$ which is referred to as the hotspot threshold. And, $\alpha$ gives flexibility to the amount of available resources as a margin factor. That is, if the amount of resources obtained in $B S 2$ is more by $\alpha$ margin than the maximum amount of available resources which the hotspot cell can hold, $B S 2$ sends hotspot release message to $B S 1$. BS1 recognizes that $B S 2$ is not the status of hotspot any more. BSI stops delay of all handovers to $B S 2$ and uses conventional handover mechanism.

In the meanwhile, it is possible that both the current serving cell and the target cell are all hotspot. Existing algorithms proposed to solve the hotspot cell problem only focus on the hotspot cell without considering the load status of neighboring cells. They only make an effort to reduce load of the hotspot cell by forcibly executing all possible handovers. These forcibly occurred handovers can not be accepted in the target cell because the target cell is also the status of hotspot. Our scheme can recognize the load status of the target cell with load information exchange before handover execution. If the current serving cell is hotspot and the target cell is also expected to the status of hotspot caused by some handover calls, the current serving cell, in our scheme, does not execute the handovers earlier, but uses conventional handover mechanism.

If the amount of load which will be increased due to handover calls is considered in advance before handover execution, cells can effectively manage their load by flexibly controlling handover time. Performance of our scheme may be significantly affected by the correctness of load information of the target cell. Typically, traffic load in a cell can be estimated by the amount of occupied resources. However, the estimation may not be exact because it does not include the amount of load expected to be added in the cell. Therefore, our scheme considers both the amount of occupied resources and the number of handover calls as the amount of load expected to be added. For load calculation procedure, we propose two new thresholds, THRES_SERVING and THRES_LOADCHECK. If the signal strength of a user $A$ is weaker than THRES_SERVING, the current serving cell requests the load information of the target cell through the backbone network. And the target cell calculates the amount of its load as the amount of available resources minus the number of users whose signal strength is stronger than THRES_LOADCHECK of the user A's target cell. Figure 2 shows how the load information is calculated. If $B S 1$ receives the report from the user (1) which includes weaker signal strength than THRES_SERVING, BSI requests the load information of the cell, $B S 2$. BS2 calculates the amount of its load by subtracting sum of users (2), (3), (4) and (5) from the amount of available resources. In our scheme, to delay handover of a user, the current serving cell should increase the transmitting power of the user to support acceptable service quality. The increase of transmitting power may lead to interference to other users served in the current serving cell and the target cell. Therefore, we should carefully select the proper threshold value, THRES_MIN in order not to interfere in other users.

In our scheme, slow handover time algorithm is used in order to delay handovers to the hotspot cell and fast handover time algorithm is used in order to execute handovers fast. In slow handover time algorithm, to delay all handovers occurred from lightly loaded cell $S$ to hotspot cell $T$, the following Equation (2) is used.

$$
\begin{aligned}
& R S S(S)<T H R E S \_S E R V I N G \\
& R S S(T)>R S S(S)+H Y S \_A C C E P T A B L E \\
& R S S(T)>T H R E S \_T A R G E T \\
& R S S(S)<T H R E S \_M I N
\end{aligned}
$$

And fast handover time algorithm uses Equation (3). That is, in case of handovers occurred from hotspot cell $S$ to lightly loaded cell $T$, handover is occurred if the following Equation (3) is satisfied.

$$
R S S(T)>R S S(S)+H Y S \_M I N
$$

Finally, in case of handovers occurred from hotspot cell $S$ to hotspot cell $T$, our scheme uses conventional handover mechanism. Conventional handover condition is equal to the following Equation (4).

$$
\begin{aligned}
& R S S(S)<T H R E S \_N O R M A L \\
& R S S(T)>R S S(S)+H Y S \_N O R M A L
\end{aligned}
$$

Two handover time algorithms organically cooperate to enhance service quality for users. Delay of handovers to hotspot cell leads to virtual expansion effect of coverage area of lightly loaded cell. And, fast execution of handovers leads to virtual shrink effect of the coverage area of the hotspot cell. Through the cooperation occurred between lightly loaded cell and hotspot cell, the shrink effect of hotspot cell is gracefully compensated by the expansion effect of lightly loaded cell. Therefore, calls can maintain connections with the network, which can support acceptable service quality for users. If the current serving cell and the target cell are all hotspot, our scheme uses conventional handover mechanism. the mechanism does not cause any change of hotspot cells' coverage area.

\section{Performance Evaluation}

In this section, we present the performance results of our scheme by simulation. We compared our scheme with soft handover area resizing in [10] and conventional handover 
mechanism. Recall that the soft handover area resizing algorithm adjusts the size of the soft handover area of hotspot cell by changing the threshold, TDROP. We used following metrics to evaluate the performance of our scheme.

1) Handover call drop rate: it is estimated as the ratio between the number of dropped calls and the total number of handover calls.

2) New call block rate: it is estimated as the ratio between the number of blocked calls and the total number of attempted calls.

3) Satisfaction rate: it is estimated as the ratio between the number of users satisfied and the total number of calls. A user is said to be satisfied if his/her call is neither blocked nor dropped.

4) The normalized number of hotspot cells: it is estimated as the number of hotspot cells normalized by the simulation time. Especially, it is only measured in 2tier environment.

\section{A. Simulation model}

We used OPNET simulator and simulations were performed in two different environments: 1tier and 2tier environment. Various models and parameters assumed in the simulation are described in Table I. As shown in Table I, We used the free space model which assumes the ideal propagation condition. The received signal strength is expressed in Equation (5). It is a function of the distance $d$ from the base station [12].

$$
R S S=m-n \log _{10}(d)+u(d)
$$

The component $u(d)$ represents shadow fading which follows log-normal distribution. The log-normal distribution is represented by the zero mean stationary Guassian random processes in $\mathrm{dB}$. We assume that all cells have the same capacity as the amount of resources which is changed during the simulation. Our 1tier and 2tier environment are set as follows.

1) Itier environment: In our 1tier environment, seven cells are deployed and the center cell has lower resources constantly than neighboring cells. The capacity of the center cell is changed from 1 to 10 and all neighboring cells have the capacity of 10 . The number of users is set to 70 .

2) 2tier environment: For our 2tier environment, nineteen cells are deployed. All cells have the same capacity of 10 and the radius of a cell is set to $0.5 \mathrm{~km}$ with considering the urban area. We performed the simulation as changing the number of users which is set to 70 initially and increases to $90,100,110$, 130 and 150. Especially, in this environment, we only studied the satisfaction rate among performance metrics.

\section{B. Simulation Results}

In the following graphs, the curves labeled as "AHT" indicate our scheme and the curves labeled as "T_DROP" indicate the soft handover area resizing in [10] which is called $T \_D R O P$ adjustment scheme in this section. And the curves labeled as "CH" indicate conventional handover mechanism which is called $\mathrm{CH}$ mechanism.
TABLE I

VARIOUS MODELS AND SIMULATION PARAMETERS

\begin{tabular}{|c||c|}
\hline Models \& Parameters & Explanation \\
\hline \hline Radio Propagation Model & Free space model \\
& $\mathrm{m}=0, \mathrm{n}=30, \mathrm{u}(\mathrm{d})-\mathrm{N}(0,8)$ \\
\hline Mobility Model & Random waypoint model \\
& $0-120 \mathrm{~km} / \mathrm{h}, 0-150 \mathrm{~s}$ \\
\hline Traffic Model & ON-OFF model \\
& ON $=120 \mathrm{~s}$, OFF $=30 \mathrm{~s}$ \\
\hline Capacity - 1tier & center cell $=1-10$ \\
& neighboring cells $=10$ \\
\hline Capacity - 2tier & all cells $=10$ \\
\hline Sampling time & $0.48 \mathrm{~s}$ \\
\hline Cell Radius & $0.5 \mathrm{~km}$ (urban area) \\
\hline Simulation time & $7200 \mathrm{~s}$ \\
\hline Parameters & H_d $=0.2, \alpha=2$ \\
& THRES_SERVING $=-74 \mathrm{~dB}$ \\
& THRES_TARGET $=-79 \mathrm{~dB}$ \\
& THRES_MIN $=-83 \mathrm{~dB}$ \\
& THRESLOADCHECK $=-77 \mathrm{~dB}$ \\
& THRES_NORMAL $=-79 \mathrm{~dB}$ \\
& HYS_ACCEPTABLE $=3 \mathrm{~dB}$ \\
& HYS_MIN $=0 \mathrm{~dB}$ \\
& HYS_NORMAL $=2 \mathrm{~dB}$ \\
\hline
\end{tabular}

First, we present the results performed in our 1tier environment. Figure 3(a) shows the results of the handover call drop rate in the center cell and Figure 3(b) shows the results in our 1 tier environment. As shown in Fig. 3(a), TDROP adjustment scheme shows lower drop rate than other schemes in the center cell, while it shows the highest drop rate among three schemes in the 1tier environment. Our scheme shows similar results with $T D R O P$ adjustment scheme and lower drop rate by $7 \%$ than $\mathrm{CH}$ mechanism in the center cell. And our scheme shows the lowest drop rate in the 1 tier environment $39 \%$ decrease over T_DROP adjustment scheme and $15 \%$ decrease over $\mathrm{CH}$ mechanism). From the results, we can conclude that our scheme can efficiently reduce traffic load of the hotspot cell like $T D R O P$ adjustment scheme and can achieve balance of load distribution in 1tier environment without aggravating load to neighboring cells.

Figure 4(a) shows the results of the new call block rate in the center cell. Results in our 1tier environment are given in Figure 4(b). In TDROP adjustment scheme, the results are similar to those of handover call drop rate. Our scheme shows higher block rate by $12 \%$ than TDROP adjustment scheme and lower block rate by $4 \%$ than $\mathrm{CH}$ mechanism in the center cell. Our scheme shows the lowest block rate in our 1 tier environment $\left(64 \%\right.$ decrease over $T \_D R O P$ adjustment scheme and $16 \%$ decrease over $\mathrm{CH}$ mechanism). The reason $T \_D R O P$ adjustment scheme has high handover call drop rate and new call block rate in 1tier environment is that it just makes an effort to reduce traffic load of the hotspot cell without considering the load of neighboring cells. Therefore, the scheme causes dropping of handover calls, then more blocking of new calls. On the other hand, our scheme shows the lowest drop rate in 1tier environment. It delays handover execution if neighboring cells are the status of hotspot. It can lead to small dropping of handover calls then, it also leads to 


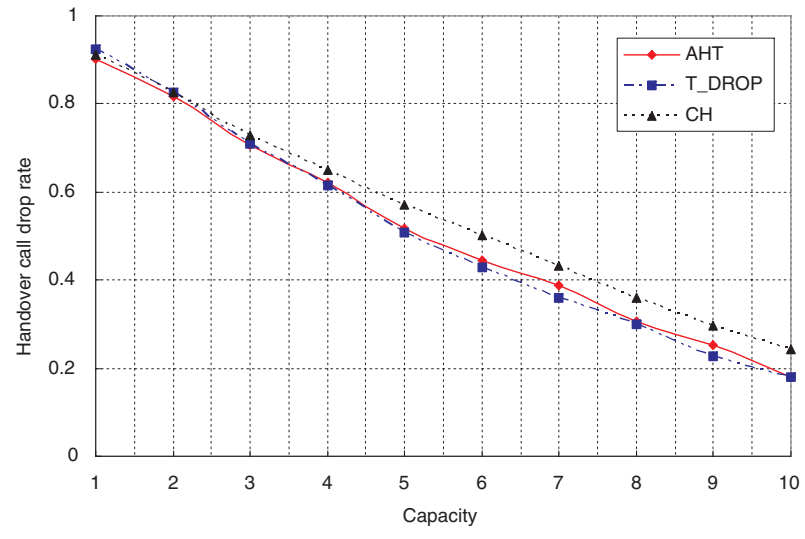

(a) Center cell

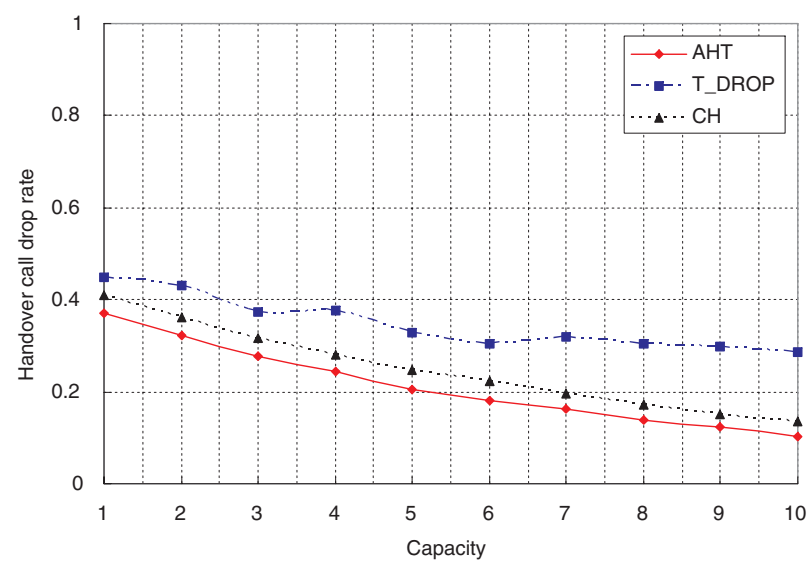

(b) 1tier environment

Fig. 3. Handover call drop rate

small blocking of new calls.

Figure 5 shows the results of the satisfaction rate in our 1tier environment. A user is said to be satisfied if his/her call is neither blocked nor dropped during the total call holding time, 120s. As shown in Fig. 5, our scheme shows higher satisfaction rate by $67 \%$ than $T \_D R O P$ adjustment scheme and by $6 \%$ than $\mathrm{CH}$ mechanism as the capacity of the center cell increases. In cellular systems, QoS(Quality of Service) guarantee for users is the important factor to determine the system performance. From this point of view, we can conclude that our scheme supports better performance than other schemes.

Now we present the results performed in our 2tier environment. Figure 6 shows the normalized number of hotspot cells in the 2tier environment. We can find that our scheme makes the smallest number of hotspot cells among three schemes. It means that our scheme can evenly distribute traffic load of cells in the network.

The satisfaction rate in our 2tier environment is given in Figure 7. Our scheme shows the highest satisfaction $(44 \%$ increase over TDROP adjustment scheme and $4 \%$ increase over $\mathrm{CH}$ mechanism)rate among three schemes. It means our

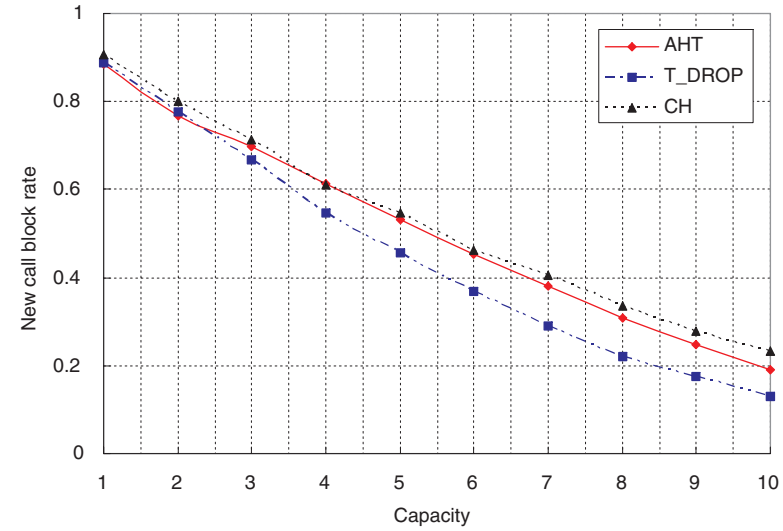

(a) Center cell

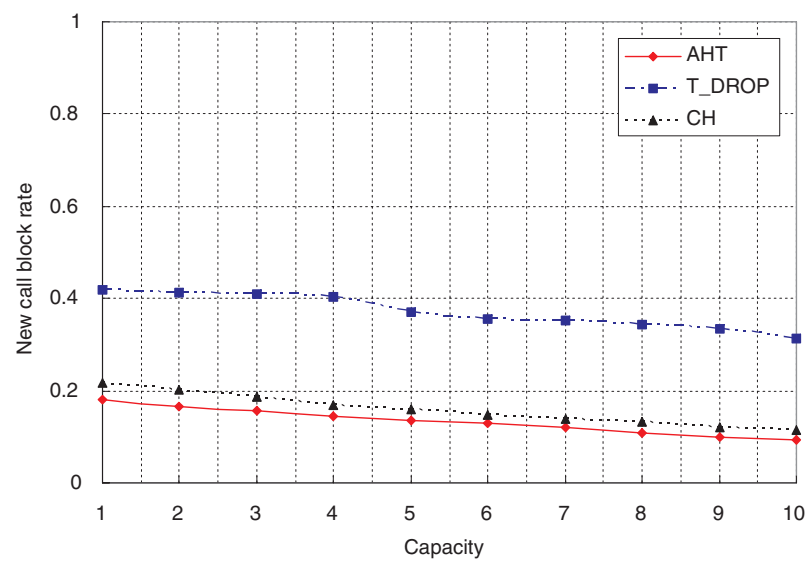

(b) 1tier environment

Fig. 4. New call block rate

scheme can efficiently use wireless resources of the current cell and neighboring cells and properly allocates to handover calls or new calls without dropping or blocking of them.

\section{CONCLUSION}

In this paper, we presented a hotspot cell management scheme based on adaptive handover time suitable for $4 \mathrm{G}$ mobile networks. Handover time is adaptively controlled according to the amount of traffic load of cells. Handovers to hotspot cell are delayed with slow handover time algorithm until the target cell obtains available resources. And hotspot cells execute possible handovers fast with fast handover time algorithm to get out of the current status and to obtain more resources. If the current serving cell and the target cell are all hotspot, our scheme uses conventional handover mechanism. As shown in the simulation results, soft handover area resizing scheme in [10] can increase the capacity of the hotspot cell. However, this scheme may cause more hotspot status at neighboring cells. It can not be the proper solution about the hotspot cell problem in $4 \mathrm{G}$ mobile networks where lots 




Fig. 5. Satisfaction rate in 1tier environment

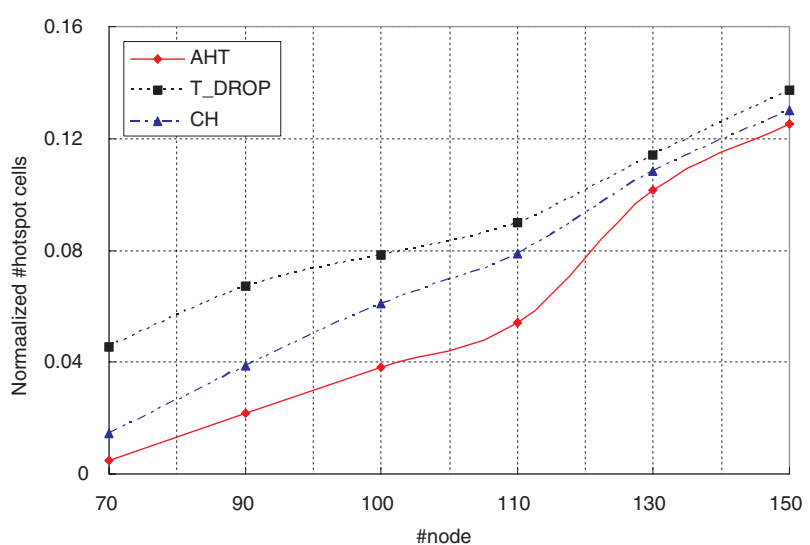

Fig. 6. The normalized number of hotspot cells in 2tier environment

of traffic flows may be highly variable. The results of our simulations allow us to draw the following conclusions.

- Handover time control based on both amount of load of the current serving cell and the target cell can effectively and flexibly manage overloaded cells in the network. Therefore, our scheme generates smaller number of hotspot cells than compared schemes.

- Service quality can be improved through hotspot cell management. Our scheme supports higher service quality than compared schemes.

In the simulation results, our scheme does not show more improved performance than conventional handover mechanism. It is caused that our scheme uses conventional handover mechanism in handovers between hotspot cells. Therefore, in the future work, we will enhance our scheme in order to achieve more balanced load distribution between hotspot cells and support higher service quality.

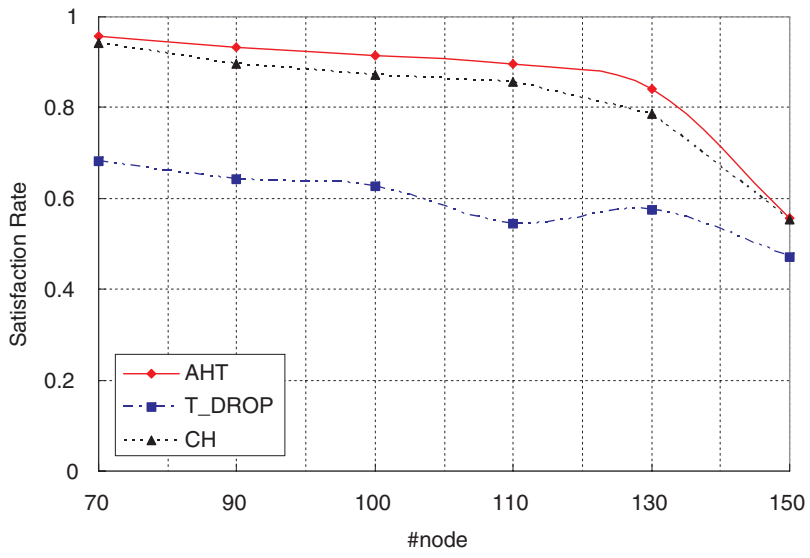

Fig. 7. Satisfaction rate in 2tier environment

\section{REFERENCES}

[1] N. Kim, H. Choi, and H. Yoon, "Seamless Handoff Scheme for $4 \mathrm{G}$ Mobile Systems based on IP and OFDM", IEEE Vehicular Technology Conference 2004-Fall, September 26-29, 2004.

[2] R. Nee, and R. Prasad, "OFDM for Wireless Multimedia Communications", Artech House, 2000.

[3] http://www.ieee802.org/16/tge

[4] http://www.ieee802.org/20

[5] J. Jobin, M. Faloutsos, S.K. Tripathi and S. V. Krishnamurthy, "Understanding the Effects of Hotspots in Wireless Cellular Networks", The 23rd Conference of the IEEE Communications Society (INFOCOM 2004), Mar, 2004

[6] D. Kim, N. Kim, and H. Yoon, "Adaptive Handoff Algorithms for Dynamic Traffic Load Distribution in 4G Mobile Networks", IEEE The 7th International Conference On Advanced Communication Technology, pp. $1269-1274$, Feb. 2005.

[7] S. Das, S. Sen and R. Jayaram, "A dynamic Load Balancing Strategy for Channel Assignment Using Selective Borrowing in Cellular Mobile Environment", Proceedings of IEEE/ACM Conference on Mobile Computing and Networking (Mobicom96), pp. 73 - 84, Nov. 1996

[8] S. Das, S. Sen P. Agrawal and R. Jayaram,"A Distributed Load Balancing Algorithm for the Hot Cell Problem in Cellular Mobile Networks", Proceedings of 6th IEEE International Symposium on High Performance Distributed Computing, pp. 254 - 263, 1997

[9] X.H. Chen, "Adaptive Traffic Load Shedding and Its Capacity Gain in CDMA Cellular Systems", IEE Pro.-Commun., Vol. 142, No. 3, pp. 186 - 192, June 1995

[10] H. Jeon, S. Hwang and S. Kwon, "A channel Assignment Scheme for Reducing Call Blocking Rate in a DS-CDMA Cellular System", Proceedings of 6th IEEE International Conference on Personal Communication, Vol. 2, pp. $637-641,1997$

[11] R. Verdone and A. Zanella, "Performance of Received Power and Traffic-Driven Handover Algorithms in Urban Cellular Networks", IEEE Wireless Communications, pp. 60 - 71, Feb. 2002

[12] P. Marichamy, S. Chakrabarti and S.L. Maskara, "Performance Evaluation of Handoff Detection Schemes", IEEE Region 10 Conference on Convergent Technologies For The Asia-Pacific, 2003 